'DV Patel, 'AS Nimbalkar, 'AR Sethi, 'AR Kungwani, 1,2SM Nimbalkar. 'Department of Pediatrics, Pramukhswami Medical College; ${ }^{2}$ Central Research Services, Charutar Arogya Mandal, Anand, India

Background and Aims To study prevalent organisms causing sepsis, their sensitivity pattern and outcome in newborn babies with culture proven sepsis.

Methods Retrospective observation of hospital records of 4 years from November 2007 to October 2011 from 276 culture positive reports with their sensitivity to the antibiotics and measured outcome of the culture proven sepsis.

Results Most common blood culture isolates in decreasing order of frequency were Klebsiella (42.4\%), Coagulase Negative Staphylococci (11.2\%), Enterobacter (9.4\%), Escherichia coli (9.1\%), Pseudomonas $(5.4 \%)$ and Acinetobacter (4.7\%). Gram negative organisms were predominant in early and late onset neonatal sepsis as well as in inborn and outborn babies. Staphylococcus aureus and Enterococci were uncommon. Candida species were isolated in early onset sepsis and in babies weighing more than $1500 \mathrm{gm}$. Most gram negative organisms were resistant to ampicillin, gentamicin and cephalosporins. Sensitivity of amikacin, levofloxacin and piperacillin-tazobactam against Gram negative organisms ranged from $25 \%$ to $75 \%$. Incidence of Methicillin Resistant Staphylococcus Aureus and Vancomycin resistant Enterococci was 33\% and 20\% respectively. Most Candida isolates were sensitive to antifungals. The most effective first line antibiotic combinations were amikacin with levofloxacin and amikacin with piperacillin-tazobactam. Overall survival rate in culture positive neonates was $43.4 \%$.

Conclusion Gram negative organisms were the most common cause of neonatal blood stream infection with high degree of resistance to commonly used first line antibiotics. These findings would help judicious selection of antibiotics when initiating them before the culture reports are available.

\section{AMPICILLINE RESISTANCE EPIDEMIOLOGY IN NEONATAL SEPSIS IN THE ERA OF INTRAPARTUM ANTIMICROBIAL PREVENTION OF EARLY-ONSET GROUP B STREPTOCOCCAL (GBS) SEPSIS}

doi:10.1136/archdischild-2012-302724.1169

${ }^{1} \mathrm{C}$ Blanckaert, ${ }^{2} \mathrm{~K}$ Husseini. ${ }^{1}$ Neonatology; ${ }^{2}$ Pediatric Intensive Care Unit, University Hospital of Poitiers, Poitiers, France

Objective To determine EOS ampicillin-resistant (AR) epidemiology and risk factors associated with ampicillin-resistant infection in newborns in the era of GBS prophylaxis.

Methods This was a retrospective analysis between 2002 and 2009, from microbiology laboratory database and hospitalization reports in one neonatal care unit. EOS was defined by a positive culture results for blood or cerebrospinal collection from infants aged $\leq 7$ days, hospitalized in the university hospital of Poitiers. Data were analyzed using Chi(2), Student's test and binary logistic regression in univariate and multivariate models.

Results EOS was identified in 30 cases. Nineteen infants $(63.3 \%)$ were preterm with GA $\leq 35$ weeks. The overall mortality rate was $23.3 \%$. Escherichia Coli (E. Coli) and GBS accounted respectively for $40 \%$ and $26.3 \%$ of the cases. Eighteen infants (62.1\%) were infected with an AR pathogen. Among E. Coli isolated, $81.8 \%$ were AR. $E$. Coli was most frequently isolated in preterm infants, $(10$ cases; $52.6 \%$ ), while $S G B$ was predominant (7 cases; $63.6 \%$ ) in term infants. EOS AR proportion was significantly higher among preterm than term infants ( $85 \%$ vs $10 \%, p \leq 0,001)$. In the AR group, GA was significantly lower, maternal age, intrapartum exposure to antibiotics and membrane rupture was higher, $(p<0.05)$. In multivariate models, GA $\leq 35 \mathrm{SA}$ was an independent predicted factor associated with AR EOS (OR 28 [95\%CI, 1.77-444.09]).
Conclusion E. Coli and AR EOS were predominant in preterm infants with GA $\leq 35$ weeks. GA $\leq 35$ weeks is an independent predicted factor of AR EOS.

\section{INCIDECE OF NEONATAL SEPSIS AND/OR MENINGITIS}

doi:10.1136/archdischild-2012-302724.1170

ER Lekic, D Dakic, L Dragas. Institut of Children Diseases, Center of Neonatology, University Clinical Center of Montenegro, Podgorica, Montenegro

Beckground and aim Infections are important cause of mortality and morbidity in the neonatal period. The purpose in this retrospective study was to identifications the bacterial microorganisms caued neonatal sepsis and/or meningitis in the all newborns hospitalized in the Center of Neonatology, during the period of 2002, 2003 and 2004 Method We used clinical, microbiological, laboratory and radiology methods.

Results 2086 infants were treated at the Center of Neonatology during the period of 2002-2004. Infants born at term (NT) were 1391, and infants born preterm (NPT) were 682. In the group of infants born at term In 528 with proven infections (omphalitis, cutaneus infections, mastitis, conjunctivitis, otitis media, infectio tracti urinary, pneumonia, diarrhea), 58 were diagnosed and treated for sepsis and/or meningitis (10.9\%). Dominant pathogens responsabile for sepsis and/or meningitis were: Staphylococcus k.n (41.3\%) i Staphylococcus Aureus (19\%), E.Coli (5, 3\%), then with equally frequency SGB, Streptococcus alfa hemolyticus rupe A, Streptococcus pneumoniae, Enterococcus, L.Monocytogenes, Klebsiella pneumoniae, (each one $1.7 \%$ ). Meningitis were proven in $16 \mathrm{TNB}$ or 27.6 per cent.

In the group of infants born preterm In $98 \mathrm{PNB}$ with proven infections (omphalitis, cutaneus infections, conjunctivitis, infectio tracti urinary, pneumonia, diarrhea), 30 PTB were diagnosed and treated for sepsis and/or meningitis (30.6\%). Dominant pathogens responsabile for sepsis and/or meningitis were: Staphylococcus aureus (26.6\%), Staphylococcus Co negative (20.0\%), Klebsiella pnaeumonia (20.0\%), Serratia marscensens (13.3\%). Meningitis were proven in $7 \mathrm{PNB}$ or $23.3 \%$ per cent.

Conclusions Preterm infants have 3 fold higher incidence of serious neonatal infections sepsis and/or meningitis.

\section{SYSTEMIC CANDIDIASIS: IMPACT OF THE SELECTIVE PROPHYLAXIS WITH FLUCONAZOLE IN RN $<1500 G R$}

doi:10.1136/archdischild-2012-302724.1171

'M Gonzalez Lopez, ${ }^{2} \mathrm{~S}$ Lacasa Maseri, 'C Gonzalez Robles, 'E Salguero Garcia 'Neonatologia; 2Pediatria, Hospital Materno Infantil, Málaga, Spain

Introduction The invasive candidiasis supposes an important problem in NICUs with a direct mortality of $15-40 \%$.

Aims and methods To describe the incidence of systemic Candida infection before and after the introduction of fluconazole prophylaxis in January 2009. Retrospective descriptive study of diagnosed patients with invasive fungal infection for 3 years in a level III hospital.

Results

Abstract 1171 Table 1

\begin{tabular}{llll}
\hline & $\mathbf{2 0 0 8}$ & $\mathbf{2 0 0 9}$ & $\mathbf{2 0 1 0}$ \\
\hline INCIDENCE $<1000 \mathrm{~g}$ & $6 / 50(12 \%)$ & $2 / 60(3.3 \%)$ & $4 / 55(7.3 \%)$ \\
INCIDENCE $<1500 \mathrm{~g}$ & $6 / 209(2.87 \%)$ & $4 / 191(2.09 \%)$ & $4 / 188(2.12 \%)$ \\
BIRTH WEIGHT<1000g & 6 & 2 & 4 \\
$>$ 1000g(<1500g) & 0 & 2 & 0 \\
GESTATIONAL AGE <28weeks & 2 & 2 & 2 \\
28-30weeks & 3 & 2 & 2 \\
$>$ 30weeks (<32weeks) & 1 & 0 & 0 \\
BLO0D CULTURES & 6 C.parapsilosis & 3 C.parapsilosis+1 & 2 C.parapsilosis+2 \\
& & C.albicans & C.albicans \\
MORTALITY & 2/6(33\%) & 0/4(0\%) & 2/4(50\%) \\
\hline
\end{tabular}

